\title{
Effects of brimonidine tartrate on $P$. acnes-induced inflammatory reaction
}

\section{So-Ra Choi ${ }^{1}$, 2 Kyung-Cheol Sohn', Young Lee1, Myung Im¹, Jeung-Hoon Lee', Seong-Jin Kim³, Chang-Deok Kim ${ }^{1 *}$}

\author{
1Department of Dermatology, School of Medicine, Chungnam National University, Daejeon, Korea. \\ 2Department of Medical Science, School of Medicine, Chungnam National University, Daejeon, Korea. \\ 3Department of Dermatology, Chonnam National University Medical School, Gwangju, Korea.
}

Brimonidine is a highly specific $\alpha 2$ adrenergic receptor agonist and acts on vascular smooth muscle to constrict blood vessels. Currently, it is used for the treatment of rosacea as a gel formulation under the brand name of Mirvaso. We experienced that Mirvaso treatment of patients with acne and rosacea resulted in alleviation of flushing as well as improvement of acne. Since it is well recognized that inflammatory reaction induced by Propionibacterium acnes (P.acnes) is critically important in the pathogenesis of acne, we speculate that brimonidine has anti-inflammatory activity in addition to its vasoconstrictive effect. In this study, we investigated the effects of brimonidine tartrate on P.acnes-induced inflammation in monocytes and keratinocytes that are importantly involved in acne pathogenesis. We examined the expression of $\alpha 2$ adrenergic receptor in both monocytes and keratinocytes. When applied to both cells, brimonidine tartrate suppressed the $P$.acnes-induced pro-inflammatory cytokine such as IL-1 $\beta$, IL-6, and IL-8 in monocytes and keratinocytes. Also, brimonidine tartrate reduced pro-inflammatory cytokine secretion by P.acnes. These results demonstrate that brimonidine has additional anti-inflammatory property besides its vasoconstrictive potential, suggesting that brimonidine is beneficial in the treatment of patients with acne and rosacea via dual action mechanisms.
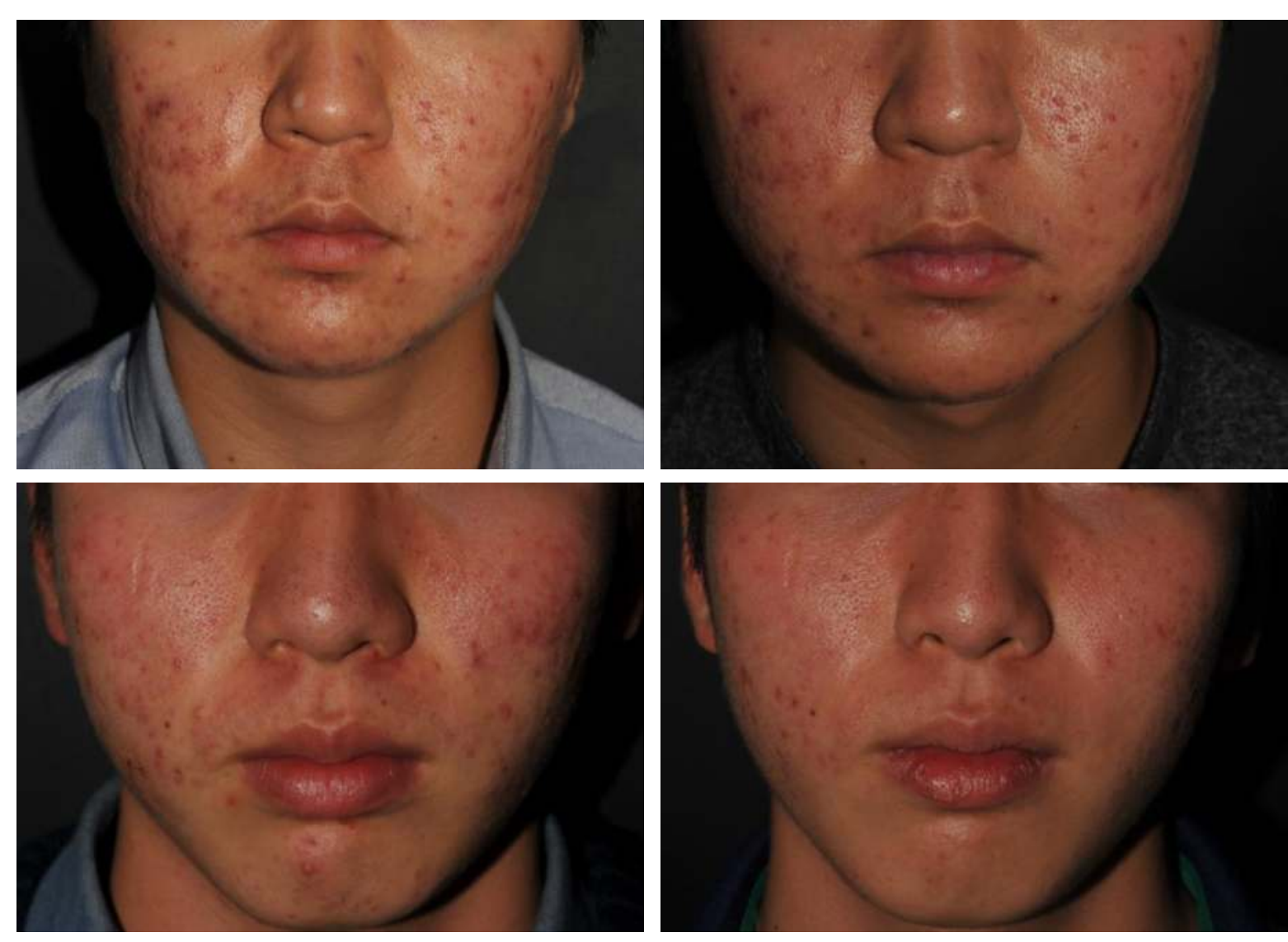

Fig. 1. Patients with acne flushing were treated with brimonidine (MIRVASO ${ }^{8}$ ) for 4 weeks. Flushing was relieved after use of brimonidine, together with reduction of inflammation.

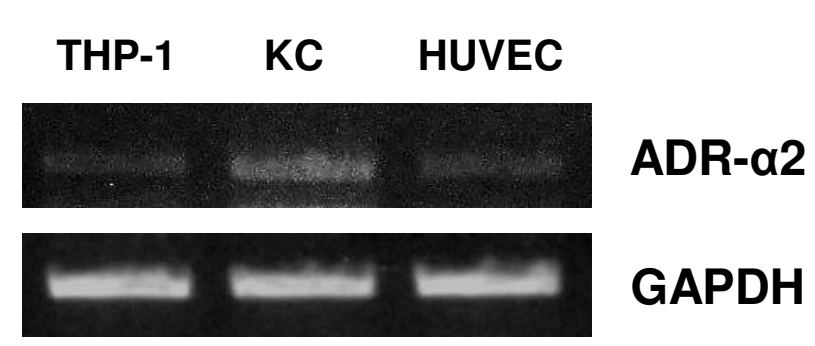

Fig. 2. Expression of adrenergic receptor a2 (ADR-a2) in monocytes (THP-1) and keratinocytes (KC). RT-PCR showed that ADR-a2 was expressed in both cells. Human umbilical vein endothelial cells (HUVEC) were used as a positive control.
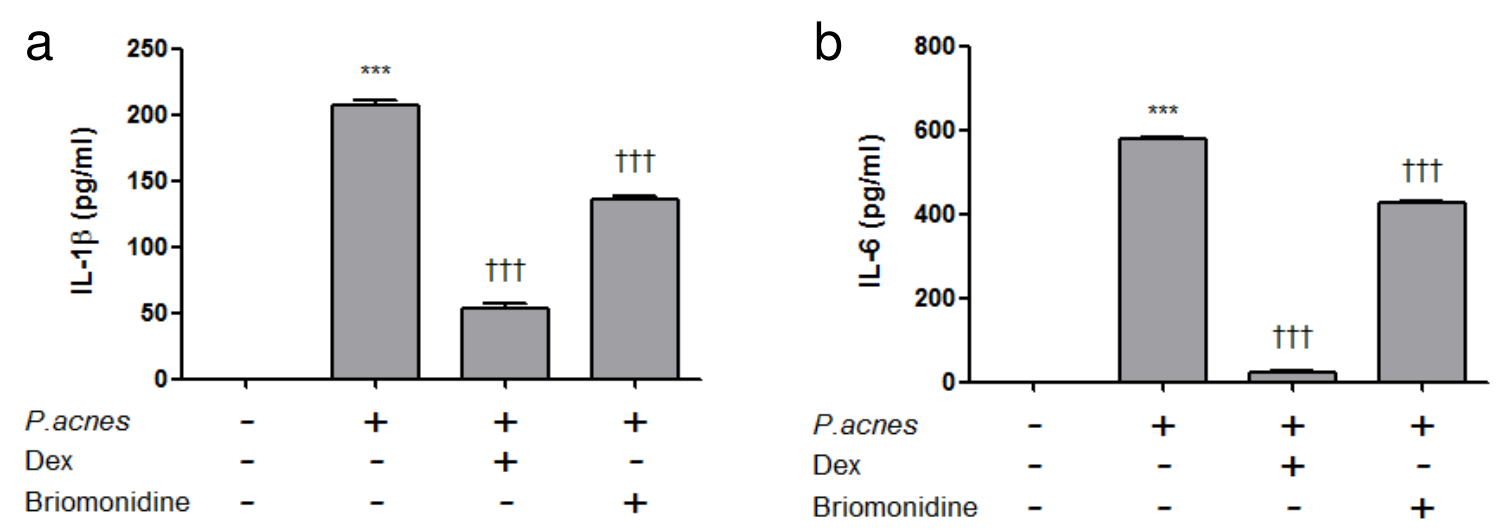

Fig. 3. Effects of brimonidine on inflammatory reaction. Monocytes (THP-1) were pre-treated with brimonidine for $1 \mathrm{~h}$, then $P$. acne was added into the cultures. After $24 \mathrm{~h}$ incubation, culture medium was collected and then cytokines were measured by ELISA. Brimonidine inhibited $P$. acne-induced secretion of cytokines significantly.
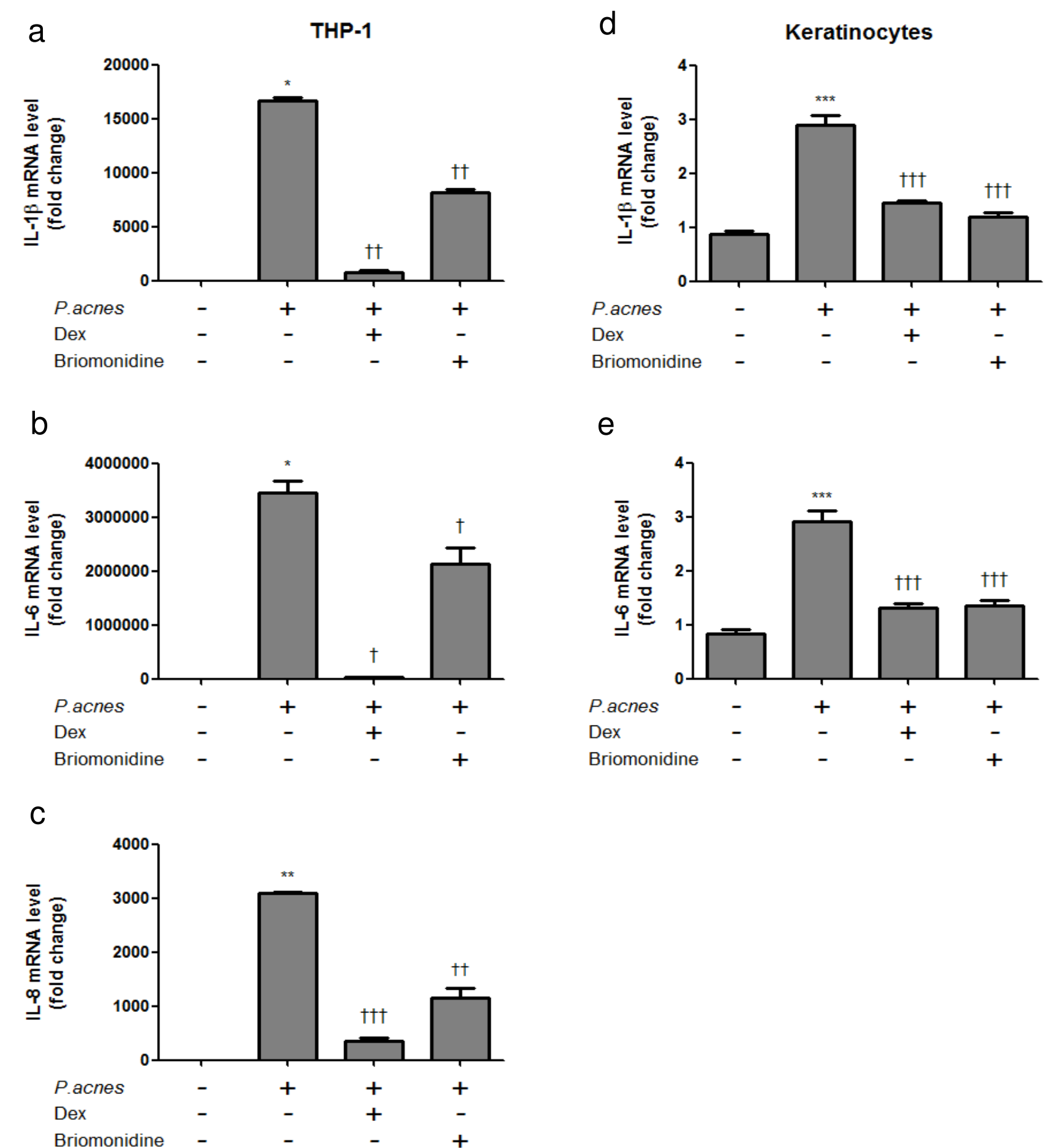

Fig. 4. Effects of brimonidine on inflammatory reaction. Monocytes (THP-1) and keratinocytes were pre-treated with brimonidine for $1 \mathrm{~h}$, then $P$. acne was added into the cultures. After $24 \mathrm{~h}$ incubation, cytokine expression was determined by real-time RT-PCR. Brimonidine inhibited $P$. acne-induced inflammatory reaction significantly in both monocytes and keratinocytes. Dexamethasone (Dex) was used as a positivie control.

\section{Conclusion}

- Brimonidine inhibits $P$. acne-induced cytokine expression in monocytes and keratinocytes.

- These results demonstrate that brimonidine has additional antiinflammatory property besides its vaso-constrictive potential, suggesting that brimonidine is beneficial in the treatment of patients with acne and rosacea via dual action mechanisms. 